beard hairs loosened. Dr. Hynds reported that he was unable to find or cultivate any growth, and saw nothing resembling blastomyces or actinomyces. When the patient was last seen after eight days' absence the verdict was reached that no treatment had been of any avail. The patient was reported to have died after rapidly increasing emaciation and gradual extension of the infection of the chin downward to the muscles of the throat and integument at the front, which left the bones bare. Death occurred about ten months after the date of first examination. Neither in this case nor in Case 1 was there any involvement of bone or periosteum.

This paper was begun nearly two years ago. The untimely death of Dr. R: W. Hynds, who did the microscopic work, together with my inability to obtain any notes of his observations, has embarrrassed me in attempting a proper report. I delayed finishing the paper in the hope, thus far futile, of getting another of those anomalous cases characterized by "granules and sour buttermilk odor." Clinically these cases bore not the slightest resemblance to the blastomycotic cases or to any of the published records of systemic, deep tissue and diffuse blastomycosis. I regret the absence of opportunity to have full histology and cultural work done on these eases, and my failure, at the time, sufficiently to appreciate them.

Characteristics were white granules, often the only contents of the cysts, deep subcutaneous abscesses, with inflammatory swelling around, hard and red, becoming Jarker and softer; and the strikingly peculiar odor. Should another case be found it would be of interest to investigate as to the probable presence and effect of the Bacillus acidi lactici.

Epithelioma, carcinoma, actinomycosis, tuberculosis, syphilis and typical blastomycosis were not suggested by any of the symptoms. If the cases were blastomycotic they were essentially as different from typical blastomycosis as granular, plus cutaneous, tuberculosis is from tuberculosis cutis verrucosa. This comparison offers the only basis for an effort to harmonize the cases I have reported with the descriptions of typical blastomycosis.

I am indebted to Dr. Oliver H. Ormsby, of Chicago, for references and suggestions.

1015 Century Building.

\section{BIRTH OF QUADRUPLETS.}

\section{HENRY A. NAVE, M.D. ARGENTINE, KANS.}

Mother's History.-Mrs. L. C. T., aged 35, American, weight 112. pounds, height $4 \mathrm{ft}$. 6 in., married twelve years, had had seven children, of whom four are still living; two were born prematurely at six and seven months respectively, and one died at the age of three months, cause of death unknown.

Present Labor. - I was called to attend the patient July 19, at 1:30 a. m. The os and cervix were well dilated and the amniotic sac was protruding. Within ten minutes the first child, a boy, was born; it seemed small, and on tying the cord I was surprised to find four umbilical cords braided together. Within fifteen minutes another boy was born, thirty minutes later a girl, half hour later another boy.

Description of Placentas.-There were two placentas, one with three cords and the other with one. They were delivered in a macerated condition.

The Children.-The length and weight of the children were as follows: First child; length 9 inches, weight about $41 / 2$ lbs.; second, length $83 / 4$ inches, weight about $31 / 2$ lbs.; third length $81 / 2$ inches, weight about $21 / 2 \mathrm{lbs}$; fourth, length 8 inches, weight about 2 lbs. In the case of the child delivered last it was necessary to resort to artificial respiration for a few minutes. The others breathed naturally. The children seemed normally developed, though small. The second child began to nurse on the second day and was apparently the only one able to swallow. The first-born child lived 24 hours; the second, 4 days; the third, 8 hours, and the fourth, 18 hours.

The mother's convalescence was uneventful except for a slight rise in temperature for a few days immediately succeeding delivery.

\section{Therapeutics}

\section{METRIO SYSTEM VS. APOTHECARIES SYSTEM.}

To the Editor:--In looking over the pages of $\Gamma_{\mathrm{HB}}$ Journal devoted to therapeutics, I have frequently been surprised at the disparity existing between the apothecaries and the metric system when both were given.

In many cases the equivalents are not even approximately correct and are extremely annoying to those who employ the metric system exclusively as well as puzzling to those who occasionally use both.

In the issue of Oct. 14, 1908, pages 1336-3\%, twelve formulas are given in which eleven are not even approximately correct and are so strikingly contradictory in the equivalent values given that a plea for a closer approximation of values seems justified.

Despite its many so-called objectionable features, there are many physicians who employ the metric system exclusively in prescribing; therefore, this loose and careless manner of giving approximate values is pernicious in its effect by encouraging slovenly methods in prescribing, and not only tends to confuse the physician but subjects the patient to extra hazard from errors in prescribing.

If we are to have equivalents given, let us have them at least approximately correct. F. A. CARMICHAEL.

The editor of the Department of Therapeutics is glad that Dr. Carmichael has brought up this subject for discussion. In the first place, the doctor does not quite understand the method with which the prescriptions which he criticises are written. If there were a stone wall between the prescriptions written in the metric system and the prescriptions written in the old system they could not be farther dissociated than they are intended now to be by the word "or." If the amounts were intended to be equivalent the sign of equality would be used. The hypothetic stone wall is represented by the word "or." "This saves writing the ingredients of the prescriptions twice. It would, of course, be difficult for a devotee of the old system to attempt to convert his thoughts and doses from that system, in any given prescription, into the metric system directly opposite.

The prescriptions in both systems are written, i. e., the liquid ones, with the idea that the dose should be represented by a teaspoonful. It is foolish to consider that a fluidram is a teaspoonful, or that the metric system equivalent to a fluidram, 4 c.c., is a teaspoonful, because they are not. It should be recognized that a teaspoonful represents 5 c.c., considerably more than a fluidram, and that by the use of the teaspoon as a measure of dosage no three fluid-ounce mixture will ever represent twenty-four doses (as it does twenty-four fluidrams), but approximately eighteen doses, of 5 c.c. each, viz.: the three ounces would represent about 90 c.c. of fluid.

The criticism is naturally then made that we should insist on the use of medicine glasses graduated to flui- 